Cedex 1, France

A. Magot

Y.Péréon

Centre de Référence

des Maladies

Neuromusculaires Rares

de l'Enfant et de l'Adulte

Nantes-Angers, Nantes,

F-44000, France

CHU de Nantes,

Laboratoire

d'Explorations

Fonctionnelles, Nantes,

F-44000, France

E. Salort-Campana

Hôpital de la Timone,

Service de Neurologie,

Centre de Référence

des maladies

Neuromusculaires

et Sclérose Latérale

Amyotrophique,

Marseille, France

F. Caillon

CHU de Nantes, Service

de radiologie, Nantes,

F-44000, France

E. Fleurence

J.Y. Mahé

Centre de Référence

des Maladies

Neuromusculaires Rares

de l'Enfant et de l'Adulte

Nantes-Angers, Nantes,

F-44000, France

Établissement de Santé

pour Enfants

et Adolescents de la région

Nantaise, Nantes, France

J. Perrier

Centre de Référence des

Maladies

Neuromusculaires Rares

de l'Enfant et de l'Adulte

Nantes-Angers, Nantes,

F-44000, France

S. Barbarot

CHU de Nantes, Clinique

dermatologique, Hôtel

Dieu, Place Alexis

Ricordeau, 44000

Nantes, France

S. Bézieau

CHU de Nantes, Service

de Génétique Médicale,

Centre de Référence

Anomalies

de Développement et

Syndromes Malformatifs

de l'inter-région

Grand-Ouest, 9, quai

Moncousu, 44093 Nantes

Cedex 1, France

\title{
Poïkilodermie héréditaire fibrosante, myopathie rétractile et fibrose pulmonaire (POIKTMP)
}

\section{Une pathologie multisystémique liée ò des mutations dans le gène FAM111B}

\author{
Sandra Mercier, Sébastien Küry, Armelle Magot, Emmanuelle Salort-Campana, \\ Florence Caillon, Emmanuelle Fleurence, Julie Perrier, Jean-Yves Mahé, Yann Péréon. \\ Sébastien Barbarot, Stéphane Bézieau
}

\section{Le syndrome POIKTMP constitue une entité multisystémique caractérisée par une} myopathie rétractile associée à des anomalies cutanées (poïkilodermie, hypotrichose), une atteinte respiratoire (syndrome restrictif, fibrose pulmonaire) et/ou des signes digestifs (insuffisance pancréatique exocrine, hépatite). Il est lié à des mutations hétérozygotes dans le gène FAM111B dont la fonction est peu connue à ce jour. Cette pathologie rare est probablement encore sous-diagnostiquée.

\section{Observation}

L'enfant R., né en 2004, est le second d'une fratrie de deux enfants issus de parents non apparentés, sans antécédent familial particulier. Il est né au terme d'une grossesse de déroulement normal avec des mensurations de naissance normales.

À l'âge d'un mois, une éruption cutanée faciale érythémateuse apparaît, déclenchée par une exposition solaire. Cette éruption évolue ensuite vers un aspect de poïkilodermie caractérisée par des lésions réticulées des joues associant des télangiectasies et une atrophie cutanée (Figure 1). Il développe également des lésions eczématiformes au niveau des extrémités. On note une hypotrichose avec des cheveux fins et épars, des sourcils et cils quasi inexistants. En revanche, ses ongles et ses dents sont sans particularité. Les parents rapportent une hypohidrose avec une intolérance à la chaleur. À l'âge de 6 ans, il présente un lymphoedème des quatre membres, prédominant aux membres inférieurs, qui s'est compliqué d'un érysipèle à deux reprises.

Dans le même temps, il développe des rétractions rapidement

Figure 1 progressives des triceps suraux conduisant à une déformation en varus des deux pieds. Il présente également un déficit musculaire distal puis proximodistal des membres inférieurs d'aggravation rapidement progressive. Il a recours au fauteuil roulant pour les déplacements extérieurs à partir de l'âge de 7 ans. Une ténotomie, effectuée à cet âge, permet à l'enfant R. de remarcher sans aide ce qui n'exclut pas un appareillage à visée orthopédique et fonctionnelle. Le déficit musculaire s'étend progressivement aux quatre membres de façon distale et proximale, sans sélectivité musculaire particulière.

L'exploration initiale, à 7 ans, met en évidence un taux de CPK normal et un tracé électromyographique myogène au niveau des membres inférieurs.
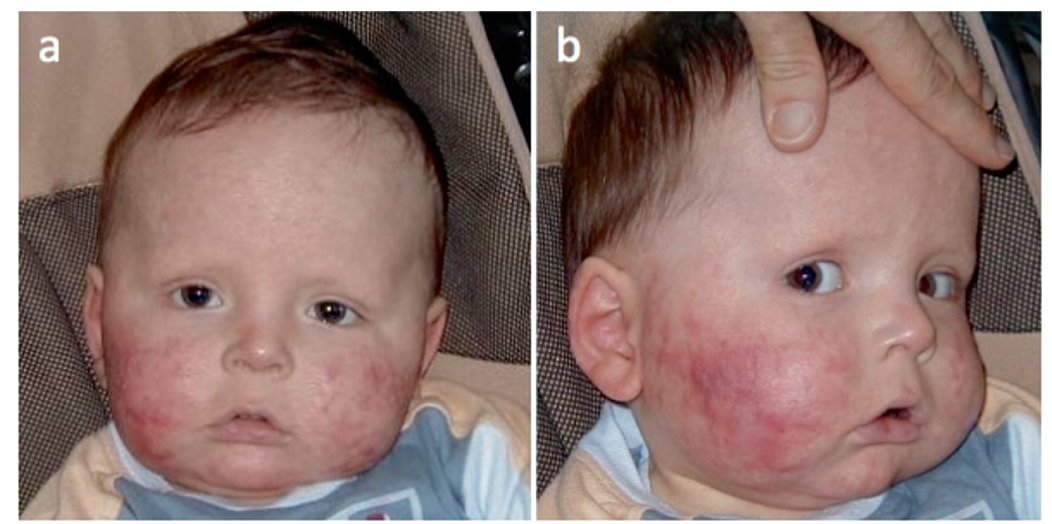

Aspect typique du visage associant poïkilodermie et hypotrichose (d'après [3]). 
L'IRM musculaire montre une infiltration adipeuse prédominant à la loge antérieure des cuisses avec épargne relative du droit fémoral et très diffuse aux jambes, épargnant relativement le tibial postérieur (Figure 2). L'électrocardiogramme est normal. Des explorations fonctionnelles respiratoires montrent un syndrome mixte restrictif et obstructif modéré chez un garçon suivi par ailleurs pour un asthme (CVF $=1,67 \mathrm{~L}(80 \%), \mathrm{VEMS}=1,32 \mathrm{~L}(77 \%)$, rapport de Tiffeneau $=79 \%$ ). À l'âge de 11 ans, un scanner thoracique ne retrouve pas de signe de fibrose pulmonaire, mais une parésie diaphragmatique gauche.

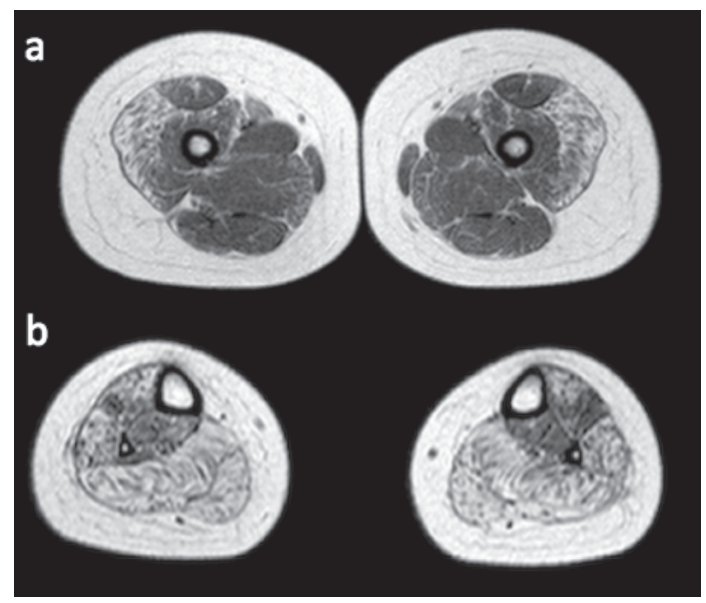

Figure 2

IRM musculaire des membres inférieurs à l'âge de 7 ans (coupes transversales : cuisses [a], jambes [b] ; séquence pondérée T1). Hyperintensités de la loge antérieure de cuisse, en particulier du muscle vaste externe, et de la loge postérieure de jambe (d'après [3]).

Une biopsie musculaire est effectuée dans le vaste externe et confirme l'infiltration adipeuse suspectée à l'imagerie (Figure $3 a, b)$. Le tissu musculaire résiduel est composé à la fois de fibres totalement préservées et de fibres dystrophiques. Aucun foyer de nécrose/régénération, aucune anomalie neurogène ne sont mis en évidence. L'aspect histologique et immunohistochimique est aspécifique dans le tissu apparemment sain. La biopsie cutanée montre un aspect fibreux, sclérodermiforme, avec une altération du réseau d'élastine formant des petits globules dans le derme papillaire (Figure $3 c, d$ ).

Nous avons alors fait le lien entre cette observation et la publication sud-africaine de Khumalo [1] qui rapportait une famille multiplex avec cinq membres atteints sur deux générations présentant une poïkilodermie, des rétractions musculaires et une fibrose pulmonaire. Le mode de transmission était autosomique dominant au vu de l'arbre généalogique. Nous avons recherché une mutation survenue de novo chez l'enfant R. par une étude d'exome en trio (étude par séquençage haut-débit de l'ADN du cas
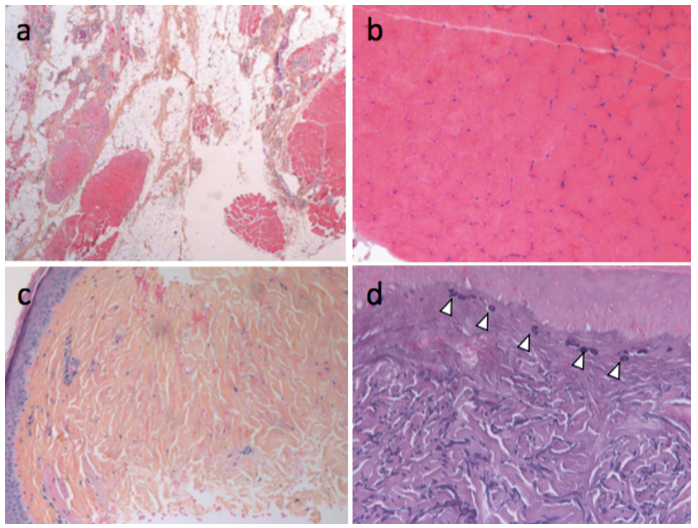

Figure 3

Biopsie musculaire (a, b) et cutanée (c, d). a : infiltration adipeuse diffuse (H\&E x 20) ; b : existence de fibres musculaires préservées $(\mathrm{H} \& \mathrm{E} \times 100)$; $\mathrm{c}$ : atrophie de l'épiderme avec aspect sclérodermiforme ( $\mathrm{H} \& \mathrm{E}, \mathrm{x} 20) ; \mathrm{d}$ : anomalies du réseau élastique avec formation de globules (flèches) (Weigert ; x150) (d'après [3])

index et des deux parents asymptomatiques). Un seul variant, c.1879A >G (p.Arg627Gly), prédit pathogène a été mis en évidence dans le gène FAM111B (NM_198947.3) dont la fonction était alors totalement inconnue. Grâce à une collaboration avec l'équipe sud-africaine, nous avons pu confirmer l'implication de ce gène dans ce nouveau syndrome et établir le diagnostic chez l'enfant R.

\section{Commentaire}

Le syndrome POIKTMP lié au gène FAM111B a été décrit par notre équipe en 2013 [2]. Depuis, nous avons recensé une cohorte de 10 familles dont huit cas sporadiques [3]. L'atteinte cutanée de type poïkilodermie survient au cours des six premiers mois de vie de manière constante dans notre série. Le deuxième signe évocateur est l'apparition de la myopathie rétractile. Les rétractions musculaires concernent classiquement les triceps suraux, mais peuvent également concerner les membres supérieurs. L'âge d'apparition des rétractions est très variable: dès l'âge de 2 ans, mais généralement vers l'âge de 6-7 ans. La ténotomie des tendons d'Achille semble avoir de bons résultats sur l'ensemble des patients opérés. Le déficit musculaire est progressif touchant à la fois les membres inférieurs et supérieurs au niveau proximal et distal. Là encore, l'atteinte est variable allant de la perte de la marche à l'âge de 3 ans pour la patiente la plus sévère de la série jusqu'à un patient adulte asymptomatique sur le plan musculaire à l'âge de 38 ans. Le tonus axial est également concerné. Certains patients présentent une scoliose modérée. L'imagerie musculaire par IRM peut renseigner sur l'atteinte musculaire et son évolutivité. Une atteinte spécifique du muscle vaste latéral a été observée 
chez le patient adulte asymptomatique. Le dosage des CPK peut être normal ou modérément élevé jusqu'à $500 \mathrm{UI} / \mathrm{L}$. L'électromyogramme peut montrer un tracé myogène ou être normal. Une biopsie musculaire, effectuée chez six patients de la série, retrouve le même aspect histologique à savoir une infiltration adipeuse majeure avec du tissu musculaire résiduel d'aspect normal ou dystrophique. Il a également été décrit des infiltrats focaux lymphocytaires ou macrophagiques sans foyer de nécrose/ régénération, ainsi qu'un aspect de fibres atrophiques avec centralisation nucléaire. Un Western blot effectué chez un patient a montré un déficit secondaire en calpaïne.

L'atteinte pulmonaire consiste généralement en un syndrome restrictif, mais certains patients ont développé, à l'âge adulte, une fibrose pulmonaire rapidement progressive en quelques années grevant le pronostic.

Le phénotype a été étendu à des atteintes digestives, en particulier à une insuffisance pancréatique exocrine. La stéatorrhée est retrouvée à l'interrogatoire. L'insuffisance pancréatique exocrine est confirmée par dosage de l'élastase fécale. Une atteinte hépatique peut également être présente avec une élévation chronique des transaminases (indépendante de l'élévation des $\mathrm{CPK}$ ), une cholestase ou une hépatomégalie selon les patients. Une famille multiplex a été publiée par Seo et al. [4] rapportant, en plus de l'atteinte cutanée, une insuffisance pancréatique exocrine chez 5/9 apparentés, ainsi qu'une atteinte hématologique chez un patient (moelle osseuse hypocellulaire). Il n'y a pas d'atteinte musculaire décrite dans cette famille, mais le phénotype n'est pas très détaillé et aucune imagerie musculaire n'a été réalisée. Dans notre série, nous rapportons aussi une atteinte hématologique à type de thrombocytopénie et d'hyperéosinophilie.

Le spectre clinique de cette pathologie va s'affiner avec le suivi des patients et le diagnostic de nouveaux patients.

Sur le plan moléculaire, cinq mutations différentes ont été identifiées. Certaines sont présentes chez plusieurs patients sans lien de parenté entre eux.
Pour les cas sporadiques, nous avons pu montrer que les mutations étaient survenues de novo lorsque le prélèvement des parents était disponible. Il s'agit de mutations faux-sens prédites pour être localisées dans le domaine enzymatique de la protéine, un domaine trypsine-like sérine protéase. La fonction de FAM111B est encore très mal connue. Un rôle dans la réplication de l'ADN est suggéré [5], ce qui serait concordant avec le rôle de RECQL4, hélicase impliquée dans le principal diagnostic différentiel, le syndrome de Rothmund-Thomson. L'atteinte musculaire semble en revanche spécifique du syndrome POIKTMP.

Nous menons actuellement des études fonctionnelles dans le modèle poisson-zèbre, sur des fibroblastes myoinduits de patients et prochainement dans un modèle murin. Nous espérons que ces travaux conduiront vers une perspective thérapeutique pour cette pathologie multisystémique, encore aujourd'hui sous-diagnostiquée malgré un phénotype reconnaissable.

Hereditary fibrosing poikiloderma with retractile myopathy, and pulmonary fibrosis: a multisystem disease associated with mutations in the FAM111B gene

\section{LIENS D'INTÉRÊT}

Les auteurs déclarent n'avoir aucun lien d'intérêt concernant les données publiées dans cet article.

\section{RÉFÉRENCES}

1. Khumalo NP, Pillay K, Beighton P, et al. Poikiloderma, tendon contracture and pulmonary fibrosis: a new autosomal dominant syndrome? Br J Dermatol 2006 ; 155 : 1057-61.

2. Mercier S, Küry S, Shaboodien G, et al. Mutations in FAM111B cause hereditary fibrosing poikiloderma with tendon contracture, myopathy, and pulmonary fibrosis. Am J Hum Genet 2013; 93 : 1100-7.

3. Mercier S, Küry S, Salort-Campana E, et al. Expanding the clinical spectrum of hereditary fibrosing poikiloderma with tendon contractures, myopathy and pulmonary fibrosis due to FAM111B mutations. Orphanet J Rare Dis 2015; 10 : 135

4. Seo A, Walsh T, Lee M, et al. FAM111B mutation is associated with inherited exocrine pancreatic dysfunction. Pancreas 2015; Oct 22. doi : 10.1097/MPA.0000000000000529.

5. Aviner R, Shenoy A, Elroy-Stein O, Geiger T. Uncovering hidden layers of cell cycle regulation through integrative multi-omic analysis. PLoS Genet 2015 ; 11 : e1005554. 